\title{
Dynamic Birefringence of Amorphous Polyolefins II. Measurements on Polymers Containing Five-Membered Ring in Main Chain
}

\author{
Tadashi InOuE ${ }^{\dagger}$, Hirotaka OKamoto ${ }^{\dagger \dagger}$, Kunihiro Osaki, \\ Teiji Kohara*, and Tadao NatsuUme* \\ Institute for Chemical Research, Kyoto University, \\ Uji, Kyoto 611, Japan \\ * Nippon Zeon Co., Ltd., Research and Development Center, \\ 1-2-1 Yako, Kawasaki-ku, Kawasaki 210, Japan
}

(Received April 13, 1995)

\begin{abstract}
Five amorphous polymers are synthesized from five membered cyclic olefins to examine the strain-induced birefringence and viscoelasticity. The complex Young's modulus and the complex strain-optical ratio are measured from the rubbery plateau to the glassy zones of viscoelasticity. The stress-optical coefficients in the rubbery plateau zone, $C_{\mathrm{R}}$, of the five polyolefins are all positive and very close to each other. This result means that the value of $C_{\mathrm{R}}$ is relatively insensitive to the details of molecular structure for this kind of polyolefins. The stress-optical coefficients in the glassy zone, $C_{\mathrm{G}}$, of the four polyolefins which do not contain benzene rings are all negative and small numbers if compared with those of polymers with the benzene rings.

KEY WORDS Viscoelasticity / Dynamic Birefringence / Stress-Optical Rule

/ Stress-Optical Coefficient / Polyolefin / Glassy Polymer /
\end{abstract}

The strain-induced birefringence of amorphous polymers relaxes complicatedly depending on temperature and polymer species. However, the birefringence is related to the stress through the simple stress-optical rule (SOR) at high temperatures or long times, i.e., in the rubbery plateau or terminal flow zones of viscoelasticity. ${ }^{1}$ This rule says that the birefringence is proportional to the stress. In oscillatory elongation with angular frequency, $\omega$, the complex strain-optical ratio, $O^{*}(\omega)=$ $O^{\prime}(\omega)+i O^{\prime}(\omega)$, is related to the complex Young's modulus, $E^{*}(\omega)=E^{\prime}(\omega)+i E^{\prime \prime}(\omega)$, as

$$
O^{*}(\omega)=C_{\mathrm{R}} E^{*}(\omega)
$$

Here $O^{*}(\omega)$ is defined as the complex ratio of the birefringence to the strain just as $E^{*}(\omega)$ is defined as the complex ratio of the stress to the strain. The coefficient $C_{\mathrm{R}}$ is called the stress-optical coefficient and is independent of $\omega$, and usually independent of temperature, over the rubbery to the terminal zones.

Another well-known relation is the photoelastic relation (PER) which is applicable to the instantaneous stress and birefringence in the glassy state. ${ }^{2}$ In oscillatory elongation, the PER corresponds to

$$
O^{\prime}(\omega)=C_{\mathrm{P}} E^{\prime}(\omega) \quad(\omega \rightarrow \infty)
$$

The coefficient $C_{\mathrm{P}}$, the photoelastic coefficient, is constant over a certain range of strain. The PER does not take account of the relaxation phenomena, or the effect of the varying frequency in the case of oscillatory deformation.

Since the coefficients, $C_{\mathrm{R}}$ and $C_{\mathrm{P}}$, are not equal to each other in general, the birefringence is not proportional to the stress in the glass-

† To whom all correspondence should be addressed.

${ }^{\dagger \dagger}$ Present address: Toyota Central Research \& Development Laboratories, Inc., 41-1, Aza Yokomichi, Oaza Nagakute, Nagakute-cho, Aichi-gun, Aichi 480-11, Japan. 
to-rubber transition zone. The birefringence has been revealed to be related to the stress with a modified stress-optical rule (MSOR) ${ }^{3}$ :

$$
\begin{gathered}
E^{*}(\omega)=E_{\mathrm{R}}^{*}(\omega)+E_{\mathrm{G}}^{*}(\omega) \\
O^{*}(\omega)=C_{\mathrm{R}} E_{\mathrm{R}}^{*}(\omega)+C_{\mathrm{G}} E_{\mathrm{G}}^{*}(\omega)
\end{gathered}
$$

where $E_{\mathbf{R}}^{*}(\omega)=E_{\mathbf{R}}^{\prime}(\omega)+i E_{\mathbf{R}}^{\prime \prime}(\omega)$ and $E_{\mathbf{G}}^{*}(\omega)=$ $E_{\mathrm{G}}^{\prime}(\omega)+i E_{\mathrm{G}}^{\prime \prime}(\omega)$ are components of the complex modulus and can be evaluated from the data of $E^{*}(\omega)$ and $O^{*}(\omega)$ if $C_{\mathrm{R}}$ and $C_{\mathrm{G}}$ are given. The coefficient $C_{\mathrm{G}}$ is defined as the ratio of the imaginary parts, $O^{\prime \prime}(\omega) / E^{\prime \prime}(\omega)$, in the glassy zone, which turned out to be a constant over a wide range of frequency for all the polymers studied so far but few exceptions. It can be easily inferred that $E_{\mathrm{R}}^{*}(\omega)$ mostly represents the behavior of the complex modulus in the low frequency region, and $E_{G}^{*}(\omega)$ that in the high frequency region, i.e., the glassy zone and the high frequency range of the glass-to-rubber transition zone.

In previous papers, we have measured the dynamic viscoelasticity and the dynamic birefringence of a number of amorphous polymers and investigated the properties of $E_{R}^{*}(\omega)$ and $E_{\mathrm{G}}^{*}(\omega)$ and the coefficients $C_{\mathrm{R}}$ and $C_{\mathrm{G}} \cdot{ }^{3-11}$ The polymers analyzed with the MSOR are; polystyrene, ${ }^{3}$ poly( $\alpha$-methyl styrene $),{ }^{4}$ a few engineering plastics $^{5}$ polyisoprene, ${ }^{6}$ and an amorphous polyolefin. ${ }^{7}$ Polyisobutylene, ${ }^{8}$ poly(1-vinyl naphthalene), ${ }^{9}$ and poly(alkyl methacrylate) $\mathbf{s}^{10}$ could not be analyzed with the MSOR. For the polymers to which the MSOR was applicable, the functions $E_{\mathrm{R}}^{*}(\omega)$ and $E_{G}^{*}(\omega)$ do not vary much with the polymer species if compared in appropriately reduced scales of frequency and modulus. The complicated $\omega$ dependence of $O^{*}$, classified in three types as will be mentioned in the discussion section, can be attributed to the combination of signs and magnitudes of $C_{\mathrm{R}}$ and $C_{\mathrm{G}}$.

Most of the polymers analyzed with the MSOR had relatively large content of benzene rings in their structures. The variation of $C_{\mathbf{R}}$ and $C_{\mathrm{G}}$ is related to the content of the benzene rings and the way how they are included in the polymer structure. ${ }^{11}$ The value of $C_{\mathrm{G}}$ is close to $3 \times 10^{-11} \mathrm{~Pa}^{-1}$ if the content is fairly high, which was the case except polyisoprene and the amorphous polyolefins. The coefficient $C_{\mathrm{R}}$ is positive or negative according to the direction of orientation of the benzene rings, parallel or perpendicular to the stretch direction, respectively. The effect of factors other than the benzene rings seems small if any. For the non-aromatic polymers studied so far, polyisoprene and the amorphous polyolefin, $C_{\mathrm{G}}$ is negative and $C_{\mathrm{R}}$ is positive and the absolute values are smaller if compared with the aromatic polymers.

In the present paper, we investigate the dynamic birefringence of five more amorphous polymers to clarify the optical properties of polymers that do not include benzene rings. The previous report on an amorphous polyolefin $^{7}$ is referred to as Part I of this series of studies.

\section{EXPERIMENTAL}

\section{Materials}

Five polymers including five-membered ring in their main chain were studied in the study: Poly(3-methyl-3-methoxycarbonyltricyclo[4.3.0.1 ${ }^{2,5}$ ]decane-7,9-diyl-ethylene) (PMMTDE); poly(dicyclo[3.3.0]octane-2,4-diyl-ethylene) (PDOE); poly(4-methyl-4-methoxycarbonylcyclopentane-1,3-diyl-ethylene) (PMMCPE); poly(4-cyclohexylcyclopentane-1,3diyl-ethylene) (PCHCPE); poly(4-phenylcyclopentane-1,3-diyl-ethylene) (PPCPE). The structures are shown in Figure 1. PPCPE includes a phenyl as a side group. Also included in the figure is the polymer of the previous study, ${ }^{7}$ poly(1-ethyl-5-methyl-octahydro-4,7methano- $H$-indene-12,3-diyl) (PEMOMID).

PMMTDE was prepared by ring-opening polymerization of 8-methyl-8-methoxy-carbonyltetracyclo[4.4.0.1 $\left.1^{2,5} \cdot 1^{7,10}\right]$-3-dodecene, followed by hydrogenation. PDOE was prepared from an endo/exo mixture of dicyclo- 
pentadiene in the same manner as above. The endo/exo ratio was 75/25. PMMCPE was synthesized from 5-methyl-5-methoxycarbonylbicyclo[2.2.1]-2-heptene in the same manner as above. PCHCPE was prepared by ring-opening polymerization of 5-phenylbicyclo-[2.2.1]-2-heptene, followed by complete hydrogenation. PPCPE was obtained by selected hydrogenation of the same intermediate product of PCHCPE.
PMMTDE

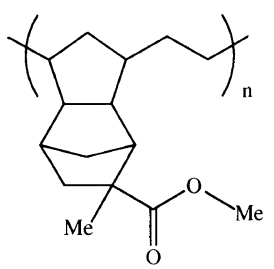

PCHCPE

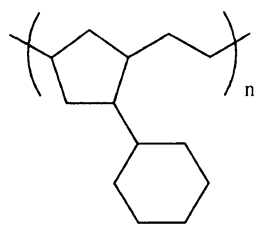

PPCPE

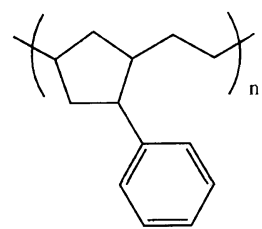

PDOE

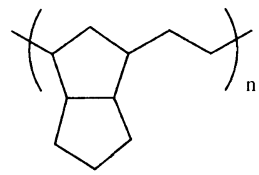

PMMCPE

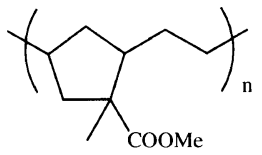

PEMOMID

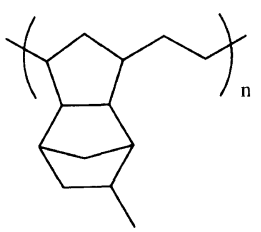

Figure 1. Structure and code of studied amorphous polyolefins.
The number average and the weight average molecular weights, $M_{n}$ and $M_{w}$ respectively, were determined by gel permeation chromatography with toluene as the eluent. Tetrahydrofuran was used as the eluent for PMMCPE which did not dissolve in toluene. The molecular weights were calibrated using standard polystyrenes. The molecular weights are shown in Table I together with the glass transition temperatures determined with differential scanning calorimetry.

\section{Measurement and Analysis}

The complex Young's modulus, $E^{*}(\omega)$, and the complex strain-optical ratio, $O^{*}(\omega)$, were measured over the range of angular frequency, $\omega$, from $6.28 \mathrm{~s}^{-1}$ to $817 \mathrm{~s}^{-1}$. The range of temperature for each sample is shown in Table I. The details of the apparatus and the procedure of measurement including the thermal history were described previously. ${ }^{7}$

For the MSOR analysis, $C_{\mathrm{R}}$ was determined as the ratio $O^{\prime}(\omega) / E^{\prime}(\omega)$ at high temperatures and low frequencies, where the ratio was independent of $T$ and $\omega$. The coefficient, $C_{\mathrm{G}}$, was determined as the ratio $O^{\prime \prime}(\omega) / E^{\prime \prime}(\omega)$ at low temperatures and high frequencies. The simultaneous equations 3 and 4, were solved for $E_{\mathrm{R}}^{*}(\omega)$ and $E_{\mathrm{G}}^{*}(\omega)$ from the determined $C_{\mathrm{R}}$ and $C_{\mathrm{G}}$ and the data of $E^{*}(\omega)$ and $O^{*}(\omega)$ at each temperature. The master curves for $E_{\mathbf{R}}^{*}(\omega)$ and $E_{\mathrm{G}}^{*}(\omega)$ were constructed with the method of reduced variables with the same procedure as stated in a previous paper. ${ }^{6}$ We have chosen the reference temperature, $T_{\mathrm{r}}$, as the temperature where $E_{\mathrm{G}}^{\prime \prime}=10^{8} \mathrm{~Pa}$ at $\omega=10 \mathrm{~s}^{-1}$, in the

Table I. Characterization of the samples

\begin{tabular}{|c|c|c|c|c|c|}
\hline Polymer code & $M_{w} / 10^{4}$ & $M_{n} / 10^{4}$ & $T_{\mathrm{g}} /{ }^{\circ} \mathrm{C}$ & $\begin{array}{c}T \text { range of } \\
\text { measurement } /{ }^{\circ} \mathrm{C}\end{array}$ & $T_{\mathrm{r}} /{ }^{\circ} \mathrm{C}$ \\
\hline PMMTDE & 4.9 & 2.1 & 163 & $205-145$ & 170 \\
\hline PDOE & 2.9 & 1.5 & 86 & $135-74$ & 90 \\
\hline PMMCPE & 4.4 & 1.8 & 49 & $80-42$ & 58 \\
\hline PCHCPE & 5.3 & 1.7 & 70 & $100-60$ & 80 \\
\hline PPCPE & 5.0 & 1.4 & 75 & $100-70$ & 80 \\
\hline
\end{tabular}


series of studies for the MSOR analysis. $T_{\mathrm{r}}$ for each sample is given in Table $I$.

\section{RESULTS}

\section{Characteristic Behavior of $E^{*}(\omega)$}

We examine the general features of the viscoelasticity and the birefringence with the master curves for $E^{*}(\omega)$ and $O^{*}(\omega)$ produced with the frequency-temperature reduction rule. The method of reduced variables with respect to $\omega$ and $T$ is not applicable in a rigorous sense to most of the amorphous polymers as pointed out in several reports. ${ }^{12}$ The breakdown of the thermo-rheological simplicity is related to the fact that the shift factor for $E^{*}(\omega)$ varied more extensively with temperature than that for $O^{*}(\omega)$ for most of the polymers in a series of preceding studies. This is also the case with the polymers in the present paper but the tendency is relatively weak. Hence the master curves are not much different from the real curves to be obtained at the reference temperature, derived by a procedure already reported in detail. ${ }^{6}$ Master curves obtained in the present study are available on request.

The master curve of $E^{*}(\omega)$ for each polymer, which is not shown here, exhibits the wellknown features of the high frequency end of the rubbery plateau zone, the glass-to-rubber transition zone, and the glassy zone, in the increasing order of the reduced frequency. The shapes of the curves are almost independent of the polymer species except the following two features.

For PMMTDE and PMMCPE, $E^{\prime \prime}(\omega)$ increases with frequency at the lowest few temperatures studied, where the polymers are well in the glassy state. This behavior, similar to that observed for poly(alkyl methacrylate)s, is probably due to the sub-dispersion in the glassy state. This conjecture may be supported by the fact that PMMTDE and PMMCPE have an ester group, which is supposed to be the origin of the sub-dispersion of poly(alkyl methacrylate)s. ${ }^{6}$ The data at these low temperatures were omitted in the following MSOR analysis. Since the sub-relaxation is well separated from the main-dispersion represented by the maximum of the loss modulus, the MSOR analysis could be carried out without any difficulty with the remaining data.

Another exception is that in the transition zone for PCHCPE the decrease of the loss modulus with decreasing frequency is relatively slow compared with the other samples; the relaxation spectrum in the glass-to-rubber transition zone is relatively broad. This feature can be attributed to the behavior of $E_{G}^{*}(\omega)$ and we will return to it in the discussion section.

\section{Characteristic Behavior of $O^{*}(\omega)$}

The frequency dependence of $O^{*}(\omega)$ is qualitatively the same as that of PEMOMID shown in Figure 6 of Part I of this series except PPCPE which contains a phenyl group.

For the major group of polymers studied here, $O^{\prime}$ is always positive, increases with increasing frequency at low frequencies, exhibits a maximum at a frequency approximately corresponding to the maximum of $E^{\prime \prime}$, and then decreases with frequency. The imaginary part, $O^{\prime \prime}$, is positive and increases with frequency at low frequencies, exhibits a maximum in the glass-to-rubber transition zone, and decreases rapidly and changes to negative at the frequency approximately corresponding to the maximum of $O^{\prime}$; then its absolute value increases rapidly, passed a maximum, and decreases gradually in the glassy zone. These features of the variation of $O^{*}(\omega)$ are qualitatively the same as those of $\operatorname{poly}(\alpha$-methyl styrene) except that the signs of the quantity are totally opposite. For PMMCPE and PCHCPE the negative values of $O^{\prime \prime}$ at high frequencies are very close to the precision limit of the measurement.

The ratios $O^{\prime} / E^{\prime}$ and $O^{\prime \prime} / E^{\prime \prime}$ are independent of the frequency and are equal to each other at low frequencies. The ratio $O^{\prime \prime} / E^{\prime \prime}$ is constant over a wide range at high frequencies for PMMTDE and PDOE. For PMMCPE and 
Birefringence of Amorphous Polyolefin II.

Table II. Coefficients representing birefringence and moduli

\begin{tabular}{lccccc}
\hline Polymer code & $C_{\mathrm{R}} / 10^{-12} \mathrm{~Pa}^{-1}$ & $C_{\mathrm{G}} / 10^{-12} \mathrm{~Pa}^{-1}$ & $C_{\mathrm{P}} / 10^{-12} \mathrm{~Pa}^{-1}$ & $E_{\mathrm{R}}^{\prime}(\infty) / \mathrm{MPa}$ & $E_{\mathrm{G}}^{\prime}(\infty) / \mathrm{MPa}$ \\
\hline PMMTDE & 1440 & -7.2 & 4.1 & 13.0 & 1650 \\
PDOE & 1950 & -9.9 & 10.3 & 15.0 & 1450 \\
PMMCPE & 1650 & -0 & 9.2 & 8.0 & 1480 \\
PCHCPE & 1200 & -5.5 & 8.6 & 16.2 & 1380 \\
PPCPE & 1270 & 25.0 & 33.3 & 10.5 & 1390 \\
PEMOMID & 1720 & -13 & 7.4 & 13.4 & 1130 \\
\hline
\end{tabular}

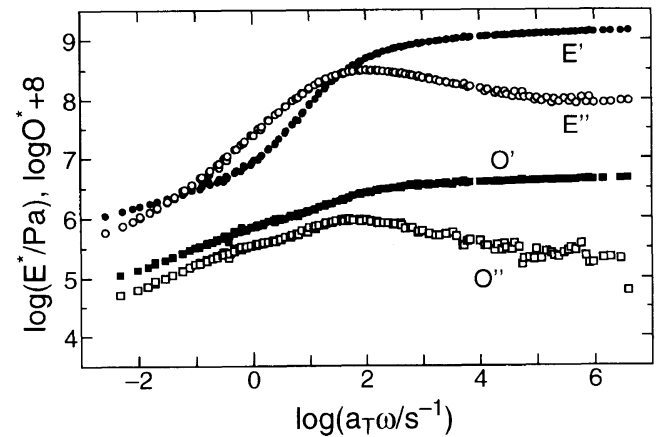

Figure 2. Frequency dependence of the complex Young's modulus and the complex strain-optical ratio for PPCPE.

PCHCPE, the magnitude of $O^{\prime \prime}$ was too small to examine the ratio quantitatively. The ratio $O^{\prime} / E^{\prime}$ levels off at high frequencies for all the studied polymers. The coefficients, $C_{\mathrm{R}}, C_{\mathrm{G}}$, and $C_{\mathrm{P}}$, are listed in Table II.

The master curves of $E^{*}$ and $O^{*}$ for PPCPE are shown in Figure 2. The qualitative features of $O^{\prime}$ and $O^{\prime \prime}$ are similar to those of Bisphenol A polycarbonate. $O^{\prime}$ is positive and increases with frequency in the transition zone and levels off in the glassy zone. $O^{\prime \prime}$ is positive, increases in the transition zone, exhibits a maximum at the frequency corresponding to the maximum of $E^{\prime \prime}$, and decreases with frequency in the glassy zone. The coefficients, $C_{\mathrm{R}}, C_{\mathrm{G}}$, and $C_{\mathrm{P}}$, are given in Table II.

On closer examination of the curve for $O^{\prime \prime}$, one may note a shoulder around $\omega=1 \mathrm{~s}^{-1}$, which has not been detected for other polymers. The frequency corresponds to the maximum of $E_{\mathrm{R}}^{\prime \prime}$ evaluated below.

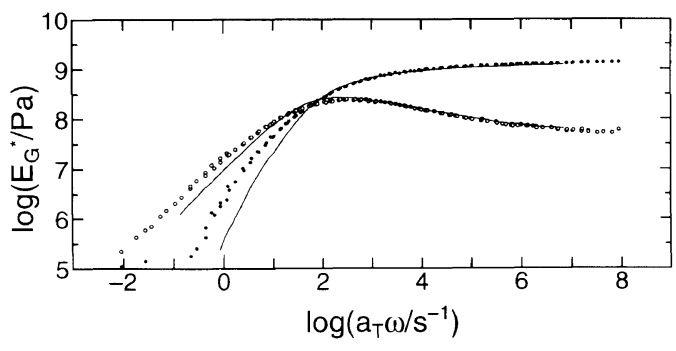

Figure 3. $E_{\mathrm{G}}^{\prime}(\omega)$ and $E_{\mathrm{G}}^{\prime \prime}(\omega)$ of PCHCPE (circles). $E_{\mathrm{G}}^{\prime}(\omega)$ and $E_{\mathrm{G}}^{\prime \prime}(\omega)$ of other samples are represented by lines for comparison.

\section{Application of the Modified Stress-Optical Rule}

The modified stress-optical rule, eq 3 and 4 , was applied to the data of $E^{*}(\omega)$ and $O^{*}(\omega)$ and the functions $E_{\mathrm{R}}^{*}(\omega)=E_{\mathrm{R}}^{\prime}(\omega)+i E_{\mathrm{R}}^{\prime \prime}(\omega)$ and $E_{\mathrm{G}}^{*}(\omega)=E_{\mathrm{G}}^{\prime}(\omega)+i E_{\mathrm{G}}^{\prime \prime}(\omega)$ were evaluated at each temperature. The method of reduced variables was applied to the results and master curves were obtained. The results for all the polymers except the sample PCHCPE are very similar to that of PEMOMID shown in Figure 2 of Part I. $^{7}$ The difference of the polymers is characterized by the values of the real part at the limit of high frequency, $E_{\mathrm{R}}^{\prime}(\infty)$ and $E_{\mathrm{G}}^{\prime}(\infty)$, respectively. These values are listed in Table II.

The $\mathrm{G}$ component of the modulus, $E_{\mathrm{G}}^{\prime}(\omega)$ and $E_{\mathrm{G}}^{\prime \prime}(\omega)$, of PCHCPE are compared with those of other samples in Figure 3. Here we choose the scales for the lines so that the maximum of $E_{\mathbf{G}}^{\prime \prime}$ overlaps that of PCHCPE. It is evident that the variation with frequency of the $E_{\mathrm{G}}^{\prime}$ and $E_{\mathrm{G}}^{\prime \prime}$ of PCHCPE is more gradual than that of the other samples represented by lines in the glass-to-rubber transition zone. 


\section{DISCUSSION}

The Stress-Optical Coefficient, $C_{\mathbf{R}}$

Table II indicates that $C_{\mathrm{R}}$ of the studied polyolefins are very close to each other: within $\pm 27 \%$ of the average value, $1.54 \times 10^{-9} \mathrm{~Pa}^{-1}$. This result implies that the value of $C_{\mathrm{R}}$ is relatively insensitive to the details of the molecular structure for the series of polyolefins. This feature is in contrast with that of the aromatic polymers, for which $C_{\mathbf{R}}$ value varies not only with the chemical composition, e.g., the content of benzene rings in the structure, but with the way how the benzene rings are involved in the molecule.

It should be noted that PPCPE, with a phenyl group sticking out of the chain, has about the same $C_{\mathrm{R}}$ value as the other amorphous polyolefins. The structure may seem similar to that of polystyrene, which has a large negative $C_{\mathrm{R}}$ value, $-5.0 \times 10^{-9} \mathrm{~Pa}^{-1}$. The large negative value of polystyrene is originated by the phenyl group which aligns perpendicularly to the stretch direction on average. The result for PPCPE evidently implies that the orientation of the phenyl group is neutral, or just in the middle of parallel and perpendicular directions with respect to the stretch direction. Indeed it is easily seen that the ring structure in the main chain restricts the phenyl group in such a direction. The $C_{\mathbf{R}}$ value would be close to that of polystyrene if the phenyl group is attached at the 2 position of the five-membered ring.

\section{The Coefficient $C_{\mathrm{G}}$}

The $C_{\mathrm{G}}$ values for the studied polymers except PPCPE are small and negative. The value for polyisoprene is about the same; $-1.1 \times 10^{-11} \mathrm{~Pa}^{-1}$. On the other hand, that for PPCPE is positive and relatively large. These observations seem to be in accord with the earlier conjecture that the $C_{\mathrm{G}}$ value is correlated with the content of the benzene rings. The relation is shown in Figure 4 for published data.

There is a fairly strong correlation. Thus for

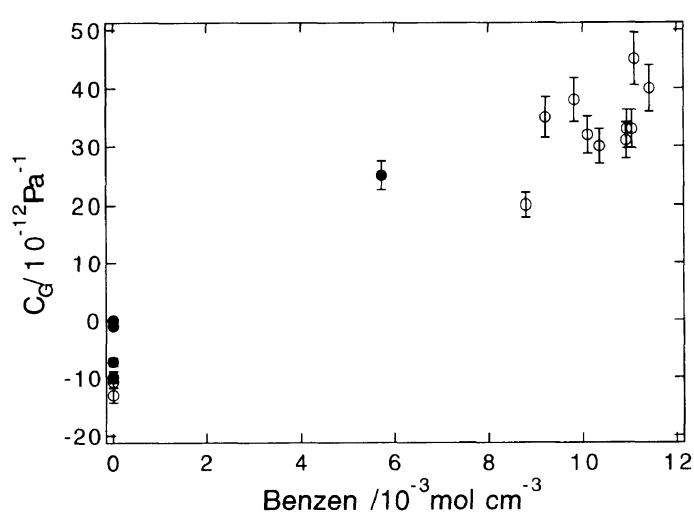

Figure 4. Benzene ring content dependence of $C_{\mathrm{G}}$. Filled and unfilled circles indicate the present and previous studies, ${ }^{3-7}$ respectively.

polymers studied so far the $C_{\mathrm{G}}$ value is mainly determined by the content of the benzene rings. The $C_{\mathrm{G}}$ value for the polymer without benzene rings is small and negative, which may be originated by the lattice effect well known for inorganic glasses. ${ }^{7}$ The benzene rings give a large positive contribution. Some other groups, not studied so far, may give large contributions and it may be interesting to study whether such contributions are simply additive or not.

\section{The Photoelastic Coefficient, $C_{\mathrm{P}}$}

The $C_{\mathrm{P}}$ values for the amorphous polyolefin derivatives without benzene rings are positive and small. The $C_{\mathbf{P}}$ value PPCPE having the phenyl group is relatively large. According to the MSOR, $C_{\mathrm{P}}$ is related to other coefficients as mentioned below. Since $C_{\mathrm{R}}$ and $C_{\mathrm{G}}$ are insensitive to the variation of polymer structure for the polyolefins, the same is true for the $C_{\mathrm{p}}$.

According to the definition of $C_{\mathrm{P}}$ and the MSOR, eq 3 and 4, one can derive the following equation.

$$
C_{\mathrm{P}}=C_{\mathrm{G}}+C_{\mathrm{R}} E_{\mathrm{R}}^{\prime}(\infty) / E_{\mathrm{G}}^{\prime}(\infty)
$$

The relation between $C_{\mathrm{R}}$ and $C_{\mathrm{P}}$ is examined in Figure 5 for literature data. ${ }^{3-7}$ The line connects the points $\left(C_{\mathrm{R}}, C_{\mathrm{P}}\right)$ and $\left(0, C_{\mathrm{G}}\right)$ for each polymer. The slope of the line should be equal to $E_{\mathrm{R}}^{\prime}(\infty) / E_{\mathrm{G}}^{\prime}(\infty)$, and in fact it is 


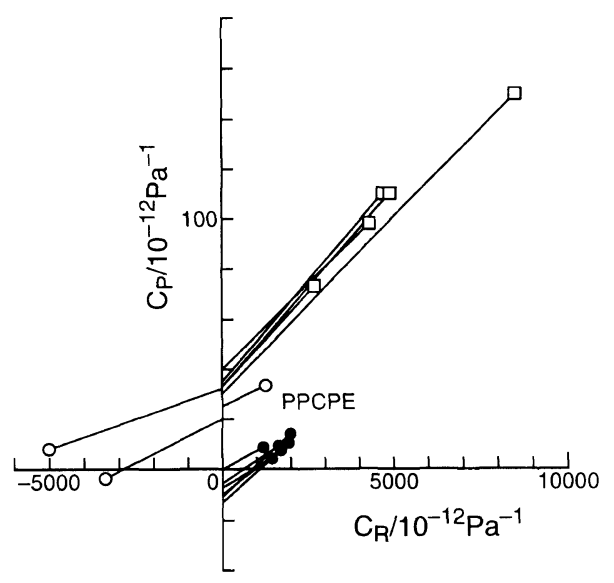

Figure 5. Relation between $C_{\mathrm{R}}$ and $C_{\mathrm{P}}$. Lines connect the points $\left(C_{\mathrm{R}}, C_{\mathrm{P}}\right)$ and $\left(0, C_{\mathrm{G}}\right)$. See the text for marks and lines.

approximately equal to the value evaluated from the data of $E_{\mathrm{R}}^{\prime}(\infty)$ and $E_{\mathrm{G}}^{\prime}(\infty)$. This equation implies that $C_{\mathrm{P}}$ is composed of contributions from $C_{\mathrm{G}}$, due to the local rotational orientation of chain unit, and $C_{\mathrm{R}}$, due to the main chain orientation. ${ }^{13}$

Figure 4 reflects the difference in the frequency dependence of $O^{*}$. If the point $\left(C_{\mathrm{R}}\right.$, $C_{\mathrm{P}}$ ) lies on the second or fourth quadrant, $O^{\prime}$ and $O^{\prime \prime}$ of the polymer change the signs. If the line connecting the points, $\left(C_{\mathrm{R}}, C_{\mathrm{P}}\right)$ and $\left(0, C_{\mathrm{G}}\right)$, crosses the $x$ axis, only $O^{\prime \prime}$ of the polymer changes its signs.

It may be worthwhile to note that there is indeed a correlation between $C_{\mathrm{R}}$ and $C_{\mathrm{P}}$ for certain groups of polymers; the group of engineering plastics having phenylene groups in the main chain (squares); the group of polyisoprene and polyolefins except PPCPE (filled circles); the group of polymers with phenyl side groups (unfilled circles), for which more data are necessary to find any systematic trend. The correlation found for polymers of similar structures may support the basic idea of the modified stress-optical rule and may be a basic result in theorizing the origin of birefringence. On the other hand, the slope of the connecting line may be related to the molecular origin of the modulus of polymer and may be useful in theorizing the origin of modulus itself. It has been conjectured that $E_{\mathrm{R}}^{\prime}(\infty)$ is related to the flexibility of the polymer chain. ${ }^{11)}$ The quantity $E_{G}^{\prime}(\infty)$ may represent a property of the conformer proposed by Matsuoka. ${ }^{14}$

\section{Features of Strain-Optical Ratio}

The qualitative features of variation of the strain-optical ratio, $O^{*}(\omega)$, can be attributed to the values of the coefficients $C_{\mathrm{R}}, C_{\mathrm{G}}$, and $C_{\mathbf{P}}$. The real part, $O^{\prime}$, and the imaginary part, $O^{\prime \prime}$, have the same sign as $C_{\mathrm{R}}$, which is positive for all the polymers studied here. If $C_{\mathrm{G}}$ and $C_{\mathrm{R}}$ have the same sign, which is the case with PPCPE and many engineering polymers, $O^{\prime}$ is an increasing function of frequency and $O^{\prime \prime}$ exhibits a maximum. The maximum of $O_{\mathbf{R}}^{\prime \prime}(\omega)$ is located at a little lower frequency than that of $O_{\mathrm{G}}^{\prime \prime}(\omega)$ but these two maxima cannot be observed separately for the engineering polymers, for which they are of the similar heights. For PPCPE the maximum of $C_{\mathrm{R}} E_{\mathrm{R}}^{\prime \prime}$ is much lower than that of $C_{\mathrm{G}} E_{\mathrm{G}}^{\prime \prime}$ and the former gives rise to the shoulder in $O^{\prime \prime}$ in Figure 2.

If the sign of $C_{\mathrm{G}}$ is opposite to that of $C_{\mathrm{R}}$, as is the case with the polyolefins, the $\boldsymbol{O}^{\prime \prime}$ changes its sign with increasing frequency. For these polymers, the last term of eq 5 , due to the chain orientation, exceeds $C_{\mathrm{G}}$ and the sign of $C_{\mathrm{P}}$ agrees with that of $C_{\mathrm{R}} ; O^{\prime}$ remains positive over the whole frequency range. This type of variation of $O^{\prime}$ and $O^{\prime \prime}$ indicates that $C_{\mathrm{P}}$ is affected largely by the chain orientation rather than by the local deformation of the chain.

If the last term of eq 5 is relatively small, the sign of $C_{\mathrm{P}}$ can be the same as that of $C_{\mathrm{G}}$. This is the case with polystyrene and $O^{\prime}$ as well as $O^{\prime \prime}$ change signs with increasing frequency.

\section{Relaxation Behavior of PCHCPE}

As pointed out in Figure 3, the increase of $E_{\mathrm{G}}^{\prime}(\omega)$ and $E_{\mathrm{G}}^{\prime \prime}(\omega)$ with frequency of PCHCPE is more gradual than other samples. This behavior is similar to that of polyisobutylene, 
for which $E_{\mathrm{G}}^{\prime \prime}$ was proportional to $\omega^{0.55}$ over a wide range of frequency in the glass-to-rubber transition zone. ${ }^{8,13}$ This behavior was attributed to the restricted rotation of the polymer units caused by the steric hindrance of two methyl groups.

On comparing the structures of the present series of polymers, a steric hindrance to the rotation of main chain methylene links may be caused by the cyclohexyl group of PCHCPE or by the phenyl group of PPCPE. The ester group of PMMCPE causes the sub-dispersion at higher frequencies and may not be significant in the transition zone. Thus it may not be impossible that the characteristic behavior of PCHCPE is caused by the hindrance of the cyclohexyl group, which is bulkier than the phenyl of PPCPE. However, it is still to be studied whether the difference of the viscoelastic behavior can be attributed to this difference of bulkiness or not.

\section{CONCLUSION}

The strain induced birefringence of the amorphous polyolefin polymers can be related to viscoelasticity through the modified stressoptical rule. The characteristic features of each polymer studied here can be represented by four parameters, $C_{\mathrm{R}}, C_{\mathrm{G}}, E_{\mathrm{R}}^{\prime}(\infty)$, and $E_{\mathrm{G}}^{\prime}(\infty)$. The stress-optical coefficient in the rubbery state, $C_{\mathrm{R}}$, for the studied polymers is found to be relatively insensitive to the details of the molecular structure. $C_{\mathrm{G}}$ is mainly determined by the benzene ring content, and is small and negative if the polymer does not have the benzene rings in its repeating structure.

Acknowledgment. This work was partially supported by a Grant-in-Aid for Scientific Research (04750737) of the Ministry of Education, Science, and Culture of Japan.

\section{REFERENCES}

1. See for example; H. Janeschitz-Kriegel, "Polymer Melt Rheology and Flow Birefringence," SpringerVerlag, Berlin, 1983.

2. D. Brewster, Trans. Roy. Soc. (London), 156(1816).

3. T. Inoue, H. Okamoto, and K. Osaki, Macromolecules, 24, 5670 (1991).

4. T. Inoue, E. J. Hwang, and K. Osaki, J. Rheol., 36, 1737 (1992).

5. E. J. Hwang, T. Inoue, and K. Osaki, Polym. Eng. Sci., 34, 135 (1994).

6. H. Okamoto, T. Inoue, and K. Osaki, J. Polym. Sci., Polym. Phys. Ed., 33, 417 (1995).

7. T. Inoue, O. Takiguchi, K. Osaki, T. Kohara, and T. Natsuume, Polym. J., 26, 133 (1994).

8. H. Okamoto, T. Inoue, and K. Osaki, J. Polym. Sci., Polym. Phys. Ed., 33, 1409 (1995).

9. E. J. Hwang, T. Inoue, K. Osaki, and A. Takano, Nihon Reoroji Gakkaishi, 22, 129 (1994).

10. O. Takiguchi, T. Inoue, K. Osaki, and A. Takano, Nihon Reoroji Gakkaishi, 23, 13 (1995).

11. K. Osaki, T. Inoue, E. J. Hwang, H. Okamoto, and O. Takiguchi, J. Non-Cryst. Solids, 172-174, 838 (1994).

12. See for example, D. Plazek, J. Phys. Chem., 69, 3480 (1965).

13. K. Osaki, O. Okamoto, T. Inoue, and E. J. Hwang, Macromolecules, 28, 3625 (1995).

14. S. Matsuoka, "Relaxation Phenomena in Polymers," Hanser Publishers, New York, N.Y., 1992. 